\title{
Whole body MRI with diffusion sequence Versus FDG- PET/CT: Correlation study on children, adolescents and young adults with Hodgkin lymphoma
}

\author{
Jose LDO Schiavon ${ }^{1 *}$, Rodrigo Regacini ${ }^{1}$, Flavio AV Luisi ${ }^{2,3}$ and Henrique M Lederman ${ }^{1,4}$ \\ ${ }^{1}$ Department of Diagnostic Imaging, Escola Paulista de Medicina, Universidade Federal de São Paulo (UNIFESP), São Paulo, Brazil \\ ${ }^{2}$ Department of Pediatrics, Division of Oncology, Escola Paulista de Medicina, Universidade Federal de São Paulo (UNIFESP), São Paulo, Brazil \\ ${ }^{3}$ Pediatric Oncologist, Pediatric Oncology Institute, GRAACC \\ ${ }^{4}$ Director, Diagnostic Imaging Center, Pediatric Oncology Institute, GRAACC
}

\begin{abstract}
On oncology patients, the risk of developing a second tumor induced by radiation is justified by the treatment benefits. However, in Hodgkin's lymphoma patients, if any effectively radiation free methods are available, they should be preferred. We correlated WB-MRI/DWI and FDG-PET/CT findings in children, adolescents and young adults with Hodgkin's lymphoma, and evaluated whether the former can be used in patient's staging and follow-up protocols as a surrogate of methods that use ionizing radiation. The patients' age at image acquisition was from 6.6 to 19.8 years (mean of $15.6 \pm 3.5$ years). 21 studies (63.6\%) were from male while 12 (36.4\%) were female patients. The WB-MRI/DWI and FDG-PET/CT imaging were performed on a mean of $7.6 \pm 4.9$ days. We found $r=0.831$ ( $<<0.001$ ) for the number of positive sites and $r=0.863$ ( $\mathrm{p}<0.001)$ for their largest diameters. Kappa concordance correlation coefficient demonstrated a sensitivity of $96 \%$ and a specificity of 95\%, with $95.2 \%$ of concordance to FDG-PET/CT and Kappa index of 0.8004 ( $\mathrm{p}<0.001$ ). WB-MRI/DWI is a safe and valuable method, with very strong Pearson correlation and almost perfect Kappa agreement with the FDG-PET/CT, which can be used as an alternative in the evaluation of children, adolescents and young adults with Hodgkin's lymphoma.
\end{abstract}

\section{Introduction}

The main therapy objective in children with Hodgkin's Lymphoma is to maximize cure rates while minimizing late toxicity, including the risk of second neoplasia. Neoplasms induced by ionizing radiation, especially in patients who may undergo to radiotherapy, should be avoided [1].

The presence and tumor extension are crucial factors to guide an excellence treatment and increase survival and cure rates. The detection of metastases and/or tumoral recurrence is "sine qua non" condition to these patients. However, whole-body exams in patients with Hodgkin's Lymphoma (HL) were discouraged from recommendations of Lugano, 2014, based on ionizing radiation exposure among other factors [2].

Computed tomography $(\mathrm{CT})$ is the main responsible for radiation exposure from unnatural source to humans [3]. However it is widely used during several tumors staging. CT combined with $18 \mathrm{~F}$-fluoro2-deoxyglucose positron emission tomography (FDG-PET/CT), is currently considered the gold standard method for the diagnosis, staging and evaluation of therapeutic response in tumors such as Hodgkin's Lymphoma [4], allowing the anatomical CT evaluation combined to functional information from the PET component.

There is a debate in the literature [5-10] on the effects of the exposure to low dose ionizing radiation from imaging studies. The risk of any procedure using ionizing radiation should be weighed against its benefits. On oncology patients, the risk of developing a second tumor is justified by the benefit of the treatment. However, in high cure rates tumors, as Hodgkin's lymphoma, if any effectively radiation free methods are available, they should be preferred.

During the last 10 years, in parallel with the development of Magnetic Resonance Imaging (MRI) sequences, techniques and devices that allow the acquisition of whole-body images from MRI (WBMRI), has been increasing its use in cancer patients and patients with multifocal diseases [4,11-19].

The use of WR-MRI with coronal short-wave inversion recovery (STIR), diffusion-imaging (DWI) sequences, and diffusion sequences with background suppression (DWIBS), has been rising due to the possibility of increasing of the MRI sensitivity and specificity, maximized by the DWI capacity, analog to PET, to localize areas of the high tumoral cellularity, developing its diagnostic performance.

High cellularity modifies Brownian motion by interacting with macromolecules and tumor cell membranes, generating DWI and DWIBS signal without promoting exposure to the inherent ionizing radiation from other imaging techniques [15-21].

*Correspondence to: José Luiz de Oliveira Schiavon, Department of Diagnostic Imaging, Rua Adalberto Ferraz 361 AP 1301, Centro, Pouso Alegre - MG, 37550-105, Brazil, Tel : +55 35-997572370; E-mail: schiavon00@gmail.com

Key words: diagnostic imaging, radiotherapy, Hodgkin's lymphoma, oncology, toxicity, ionizing radiation

Received: August 30, 2019; Accepted: September 10, 2019; Published: September 13, 2019 

lymphoma

We correlated WB-MRI/DWI and FDG-PET/CT findings in children, adolescents and young adults with Hodgkin's lymphoma, and evaluated whether the former can be used in patient's staging and follow-up protocols as a surrogate of methods that use ionizing radiation.

\section{Methods}

This study was conducted by the Diagnostic Imaging Department of the Federal University of São Paulo (UNIFESP) at the Pediatric Oncology Institute of the Adolescent and Children with Cancer Support Group (IOP / GRAACC), after being accepted by the Research Ethics Committee of UNIFESP (1439/2018).

We selected images of patients with Hodgkin's Lymphoma (HL), surveyed by the Hospital Information System (HIS) during the period 2015-2017, and submitted to the following inclusion and exclusion criteria.

Were included in this study patients of up to 20-years old whose presented FDG-PET/CT images performed for Hodgkin's lymphoma evaluation, as well as a WB-MRI/DWI, stored in the institution's PACS and acquired in a period of no more than 21 days from the first study, from 2015 to 2017, with confirmed histopathological diagnosis of HL.

Patients who did not have a confirmed diagnosis by hispopathological analysis, or incomplete exams stored to PACS, and those who underwent for both examinations on a more period of more than 21 days between the WB-MRI/DWI and FDG-PET/CT acquisitions were excluded.

After the patients' electronic data were submitted to these criteria, the hospital registry of the eligible patients' records was coded in ascending ordinal numerical order, in order to preserve the identity of the patients. Their images were then anonymized for the interpretation.

The FDG-PET/CT images were obtained from several institutions, according to the internal protocols of these exam providers, after requested by the assistant medical team, based on the HL protocols, and made available to the IOP/GRAACC HIS/PACS and research.

The interpretation of the FDG-PET/CT images for this study was performed independently from WB-MRI/DWI, at different and random times.

The interpretation data found by this study were later verified with the final report provided by the FDG-PET/CT exam provider, so that possible relevant discrepancies in the clinical conduct of the patient could be informed to the attending physician. Only the findings reported by the physicians from this study were considered.

All the exams were reviewed by the two radiologists, with 40 and 8 years of experience in pediatric radiology. Differences of interpretation among the evaluators were resolved in consensus, before access to any other data present in the electronic medical records.

Findings considered positive by the WB-MRI/DWI reviewers were then tabulated for the comparative analysis in a structured report, that allow counting of affected sites and also, the largest dimension, in centimeters, of lymph node clusters. The FDG-PET/CT findings were then given the same tabulations.

WB-MRI/DWI exams were obtained in a $1.5 \mathrm{~T}$ device (Achieva ${ }^{\circ}$, Philips Healthcare, Best, The Netherlands) with integrated body coil and a maximum gradient power of $28 \mathrm{mT} / \mathrm{m}$.
The patients were examined in the supine position, with arms to the side, and images acquired independent of respiratory movements.

The T2 STIR sequence was performed, without administration of paramagnetic, coronal contrast, following the following parameters:

- Repetition time (TR) / echo time (TE): 3133 / 30ms, inversion time (TI): $160 \mathrm{~ms}$, matrix: 220x320, field of view (FOV): $480 \mathrm{~mm}^{2}$, NEX: 4, thickness of: $5 \mathrm{~mm}$, interval: $1 \mathrm{~mm}$, duration: 35-40 min.

Subsequently, the diffusion weighted image with background suppression (DWIBS) was obtained in the axial plane, following the following parameters:

- Value b: 0 and $800 \mathrm{~s} / \mathrm{mm}^{2}$; TR / TE: 8263 / $70 \mathrm{~ms}$, TI: $150 \mathrm{~ms}$, matrix: $176 \times 115$, FOV: $530 \mathrm{~mm}^{2}$; NEX: 4, thickness: $6 \mathrm{~mm}$; range: -1 $\mathrm{mm}$; duration 10-15 min.

The sequences were post-processed and reconstructed in full body with the Mobiview ${ }^{\circledR}$ software.

The obtained images were evaluated by the following criteria to be classified as abnormal.

Presence of anomalous hyper signal in the STIR sequence, with DWIBS abnormal signal, associated with the morphology or dimensions considered unusual for topography. The lymph node analysis included

Table 1. Positive HL involvement count in lymph nodes or extranodal sites

\begin{tabular}{|c|c|c|c|c|}
\hline \multicolumn{5}{|c|}{ Number of positive HL involvement sites } \\
\hline Phase & Patient & Study & FDG-PET/CT & WB-MRI/DWI \\
\hline Follow-up & 1 & 1 & 4 & 4 \\
\hline Follow-up & 2 & 2 & 6 & 6 \\
\hline Follow-up & 3 & 3 & 0 & 4 \\
\hline Follow-up & 3 & 4 & 0 & 0 \\
\hline Follow-up & 3 & 5 & 0 & 0 \\
\hline Staging & 4 & 6 & 5 & 5 \\
\hline Follow-up & 5 & 7 & 0 & 3 \\
\hline Follow-up & 6 & 8 & 0 & 1 \\
\hline Staging & 7 & 9 & 8 & 5 \\
\hline Follow-up & 8 & 10 & 3 & 4 \\
\hline Follow-up & 9 & 11 & 0 & 0 \\
\hline Staging & 10 & 12 & 6 & 10 \\
\hline Follow-up & 10 & 13 & 0 & 0 \\
\hline Follow-up & 10 & 14 & 0 & 0 \\
\hline Follow-up & 10 & 15 & 0 & 0 \\
\hline Follow-up & 11 & 16 & 0 & 8 \\
\hline Staging & 12 & 17 & 1 & 1 \\
\hline Follow-up & 13 & 18 & 1 & 1 \\
\hline Staging & 14 & 19 & 6 & 6 \\
\hline Follow-up & 14 & 20 & 6 & 6 \\
\hline Staging & 15 & 21 & 3 & 3 \\
\hline Follow-up & 15 & 22 & 0 & 0 \\
\hline Follow-up & 15 & 23 & 0 & 0 \\
\hline Follow-up & 15 & 24 & 0 & 0 \\
\hline Follow-up & 16 & 25 & 0 & 0 \\
\hline Staging & 17 & 26 & 11 & 11 \\
\hline Follow-up & 18 & 27 & 3 & 5 \\
\hline Follow-up & 19 & 28 & 3 & 5 \\
\hline Staging & 20 & 29 & 7 & 9 \\
\hline Follow-up & 20 & 30 & 2 & 2 \\
\hline Follow-up & 20 & 31 & 0 & 0 \\
\hline Follow-up & 20 & 32 & 0 & 0 \\
\hline Follow-up & 20 & 33 & 0 & 0 \\
\hline TOTAL & & & 75 & 99 \\
\hline
\end{tabular}


Schiavon JL (2019) Whole body MRI with diffusion sequence Versus FDG-PET/CT: Correlation study on children, adolescents and young adults with Hodgkin lymphoma

Table 2. Measurement of larger lymph node clusters considered positive for HL

\begin{tabular}{|c|c|c|c|c|}
\hline \multicolumn{5}{|c|}{ Larger dimensions of HL lymph nodes (in $\mathrm{cm}$ ) } \\
\hline Phase & Patient & Study & FDG-PET/CT & WB-MRI/DWI \\
\hline Follow-up & 1 & 1 & 12 & 13.1 \\
\hline Follow-up & 2 & 2 & 10 & 15 \\
\hline Follow-up & 3 & 3 & 0 & 10 \\
\hline Follow-up & 3 & 4 & 0 & 0 \\
\hline Follow-up & 3 & 5 & 0 & 0 \\
\hline Staging & 4 & 6 & 16.5 & 17.2 \\
\hline Follow-up & 5 & 7 & 0 & 13 \\
\hline Follow-up & 6 & 8 & 0 & 5 \\
\hline Staging & 7 & 9 & 11.3 & 10 \\
\hline Follow-up & 8 & 10 & 2.5 & 5.1 \\
\hline Follow-up & 9 & 11 & 0 & 0 \\
\hline Staging & 10 & 12 & 18 & 20 \\
\hline Follow-up & 10 & 13 & 0 & 0 \\
\hline Follow-up & 10 & 14 & 0 & 0 \\
\hline Follow-up & 10 & 15 & 0 & 0 \\
\hline Follow-up & 11 & 16 & 0 & 10 \\
\hline Staging & 12 & 17 & 11 & 12.6 \\
\hline Follow-up & 13 & 18 & 4.3 & 4.3 \\
\hline Staging & 14 & 19 & 7.8 & 5.9 \\
\hline Follow-up & 14 & 20 & 7.8 & 5.9 \\
\hline Staging & 15 & 21 & 15 & 16.9 \\
\hline Follow-up & 15 & 22 & 0 & 0 \\
\hline Follow-up & 15 & 23 & 0 & 0 \\
\hline Follow-up & 15 & 24 & 0 & 0 \\
\hline Follow-up & 16 & 25 & 0 & 0 \\
\hline Staging & 17 & 26 & 21.3 & 21.3 \\
\hline Follow-up & 18 & 27 & 3.5 & 4.8 \\
\hline Follow-up & 19 & 28 & 3.5 & 4.8 \\
\hline Staging & 20 & 29 & 9 & 9 \\
\hline Follow-up & 20 & 30 & 9 & 8 \\
\hline Follow-up & 20 & 31 & 0 & 0 \\
\hline Follow-up & 20 & 32 & 0 & 0 \\
\hline Follow-up & 20 & 33 & 0 & 0 \\
\hline
\end{tabular}

Table 3. Comparative analysis of abnormal signal suggestive of HL involvement on WB$\mathrm{MRI} / \mathrm{DWI}$ and FDG-PET/CT

\begin{tabular}{|c|c|c|c|c|}
\hline $\begin{array}{c}\text { WB-MRI/DWI } \\
\text { HL involvement } \\
\text { sites }\end{array}$ & \multicolumn{2}{|c|}{$\begin{array}{c}\text { FDG-PET/CT } \\
\text { HL involvement sites }\end{array}$} & \\
\hline & & YES & NO & TOTAL \\
\hline YES & & 72 & 27 & 99 \\
\hline NO & & 3 & 526 & 529 \\
\hline TOTAL & & 75 & 553 & 628 \\
\hline Concordance & $95,22 \%$ & Kappa & 0,8004 & $\mathrm{p}<0,001$ \\
\hline
\end{tabular}

a dimension larger than $1.0 \mathrm{~cm}$ in its smaller axial plane diameter as involvement criteria.

All data obtained were then submitted to statistical analysis by commercial software SPSS ${ }^{\circledR}$ for Windows version 17.0 (Tables 1 and 2).

Descriptive analyzes such as the mean patients' age, gender percentile and the examination time (staging or follow-up) were tabulated.

Pearson's correlation test was selected after assessing the distribution data curve for the sites' number and largest lymph node clusters diameter in each method. A comparative table (Table 3) of Kappa concordance coefficient was also generated considering FDG$\mathrm{PET} / \mathrm{CT}$ as gold standard.
The significance level adopted for all tests was $\mathrm{p}<0.05$.

\section{Results}

72 studies which met the initial prerequisites with both FDGPET/CT and WB-MRI/DWI were found. 39 were excluded since 23 have undergone examinations in a time interval of more than 21 days, and 16 that had incomplete MRI sequences.

The total of 33 studies from 20 different patients was included. $8(24.2 \%)$ were acquired during staging and 25 on disease follow-up $(75.8 \%)$.

The patients' age at image acquisition was from 6.6 to 19.8 years (mean of $15.6 \pm 3.5$ years). 21 studies (63.6\%) were from male while 12 (36.4\%) were female patients. The WB-MRI/DWI and FDG-PET/CT imaging were performed on a mean of $7.6 \pm 4.9$ days.

A total of 75 sites were considered positive on FDG-PET/CT, while WB-MRI/DWI observed 99 positive sites (Table 1).

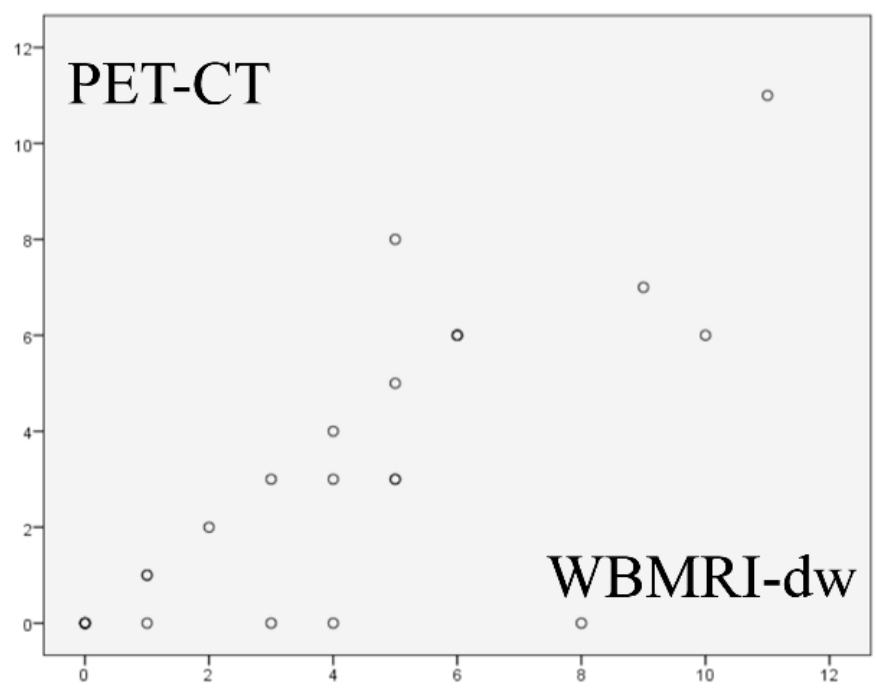

Graph 1. Distribution of body sites considered positive for HL involvement

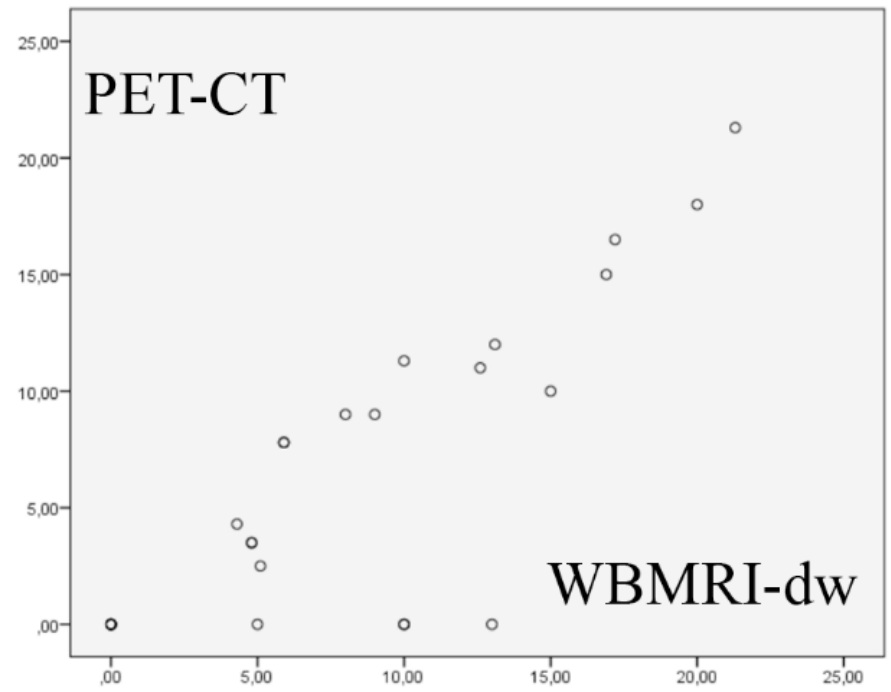

Graph 2. Distribution of the largest dimensions of the lymph node clusters considered affected by HL 

lymphoma

The largest lymph node dimensions considered positive for HL was $21.3 \mathrm{~cm}$ in both methods, with a mean of $4.9 \pm 6.4 \mathrm{~cm}$ in FDG-PET/CT and $6.4 \pm 6.7 \mathrm{~cm}$ in WB-MRI / DWI (Table 2).

Findings were then correlated by the Pearson Correlation method (-1 to 1 ) after the evaluation of the distribution curve (Graphs 1 and 2).

We found $\mathrm{r}=0.831(\mathrm{p}<0.001)$ for the number of positive sites (Graph 1) and $\mathrm{r}=0.863(\mathrm{p}<0.001)$ for their largest diameters $($ Graph 2$)$.

A comparative table for Kappa concordance correlation coefficient with FDG-PET/CT as gold standard demonstrated a sensitivity of $96 \%$ and a specificity of $95 \%$, with $95.2 \%$ of concordance and Kappa index of 0.8004 ( $\mathrm{p}<0.001)$.

\section{Discussion}

FDG-PET/CT images infer cellularity from tumoral lesions by its glucose consumption that can be assessed from its standard uptake value (SUV). This study did not use neither the SUV nor the apparent diffusion coefficient (ADC) for DWI restriction measuring, as both can vary from different acquisition sites, and a quantitative index does not influence in HL conduction.

The development of MRI techniques, especially water-sensitive series, such as STIR and DWIBS, allowed a good evolution in the evaluation of inflammatory sites, as DWI sequences create images demonstrating the restriction to the random water movement by the increase of cellularity and membrane changes, which promote a phasic restricted dispersion of the proton spin.

Huang et al. in 2009 estimated that the effective dose of FDGPET/CT ranged from 13.45 to $31.91 \mathrm{mSv}$, equivalent to up to 320 chest radiographs for a single exam, associated with an assigned risk of life-time cancer development estimated between $2.31 \%$ and $5.14 \%$ for American women aged 20 at the time of exposure, and $1.63 \%$ to $3.23 \%$ for American men in the same age group [11]. Another study published in 2016 showed that, even on modern devices, the effective exposure dose for FDG-PET/CT remained in the order of $5.89 \pm 1.46$ $\mathrm{mSv}$ for the PET the radioisotope (18F-fluoro- 2-deoxyglucose) and $6.26 \pm 3.06 \mathrm{mSv}$ from its CT component [12].

Magnetic Resonance Imaging (MRI) exempts patients from any risk related to ionizing radiation. The advent of new acquisition sequences, as DWI, first described under MRI in 1965 by Steiskel and Tanner [13], allows us to determine the tumoral therapeutic response by its membrane and cellularity modifications.

The change of Brownian motion, applied to the human body, by diffusion, effectively represents the movement of free water in the intracellular and extracellular spaces [14]. It can be used as an analog to the consumption of the glucose characterized by FDG-PET/CT, with viability already described in some pediatric patients with Hodgkin's lymphoma $[15,16]$.

A meta-analysis of 1239 adult patients comparing WB-MRI/ DWI and FDG-PET/CT showed similarity between those methods, however, this study is a sum of several small heterogeneous studies in its methodology of an adult population, and of different neoplasms [22].

In HL the use of FDG-PET/CT, or only CT when the first is not available, is recommended by protocols for staging and follow-up $[16,23,24]$, but WB-MRI/DWI has been proving comparable ability to detect involvement by HL, with demonstrated agreement [15-16] sometimes put in questionable [25].
Hodgkin's lymphomas modified Ann Arbor staging classification [26] and Lugano [2] are based, in addition to other factors, on the tumoral involvement sites, conferring the imaging studies a fundamental role in the therapeutic protocol $[4,27]$.

The evaluation of WB-MRI/DWI staging and follow-up on Hodgkin's lymphoma protocols in children and adolescents requires publications [27-33], in order obtain sufficient data to establish WB$\mathrm{MRI} / \mathrm{DWI}$ as a reliable alternative to FDG-PET/CT.

The main findings in WB-MRI/DWI related to Hodgkin's lymphoma also characterized in our study are: hyper signal mass in STIR (Figure 1A) and hyper cellular DWI abnormal signal (Figure 1B). Hodgkin's Lymphoma FDG-PET/CT findings were mass with high 18F-FDG uptake, as shown in Figures 2A and 2B.

Studies in adults and children [4,15-19] determined that both methods have similar accuracy, and additional sequences that can be performed during MRI scans can increase the its diagnostic performance [22].

WB-MRI/DWI was also considered as reliable as other conventional studies in the initial staging of small cell tumors by several authors and presents promising results in positive findings in the study of bone marrow involvement in Hodgkin's lymphoma compared to FDG-PET/CT [4,15-19].

Our study, in children, adolescents and young adults, found similar findings among those methods, with a very strong Pearson correlation

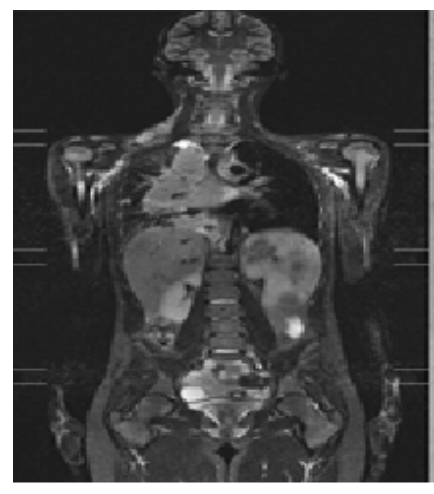

A

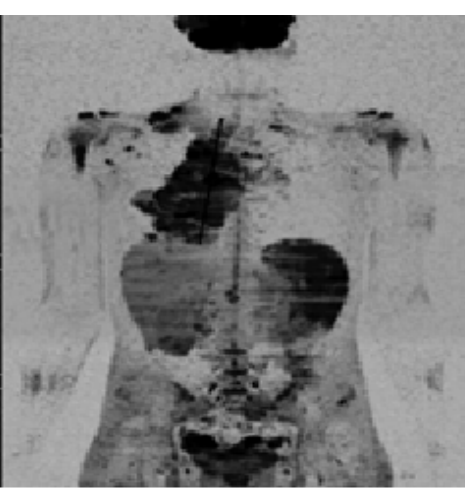

B
Figure 1. (A) WB-MRI/DWI coronal STIR image, demonstrating mass in the supraclavicular, mediastinal, bone marrow, right humerus, splenic and pelvis sites, with greater lesion in the right mediastinum. (B) WB-MRI DWIBS MIP reconstruction measuring the largest positive site

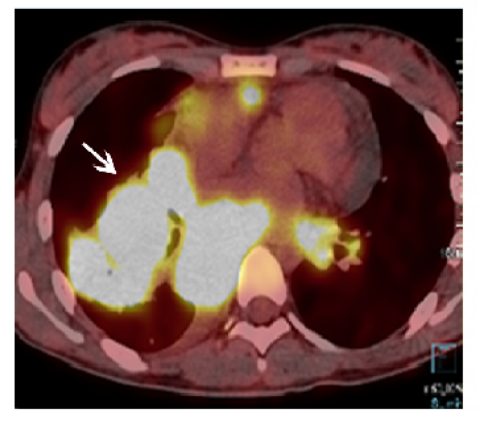

A

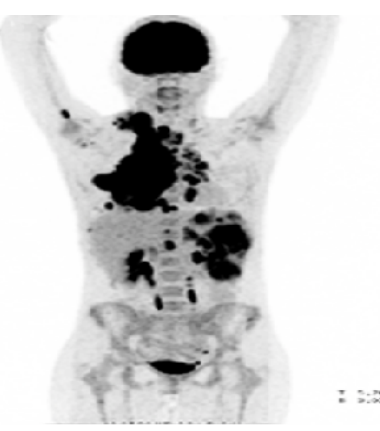

B
Figure 2. (A) Axial FDG-PET/CT fusion at the mid-thorax demonstration mediastinal mass with high SUV on the right (arrow). (B) PET image obtained from the FDG-PET/CT examination with 3D-MIP total body reconstruction 

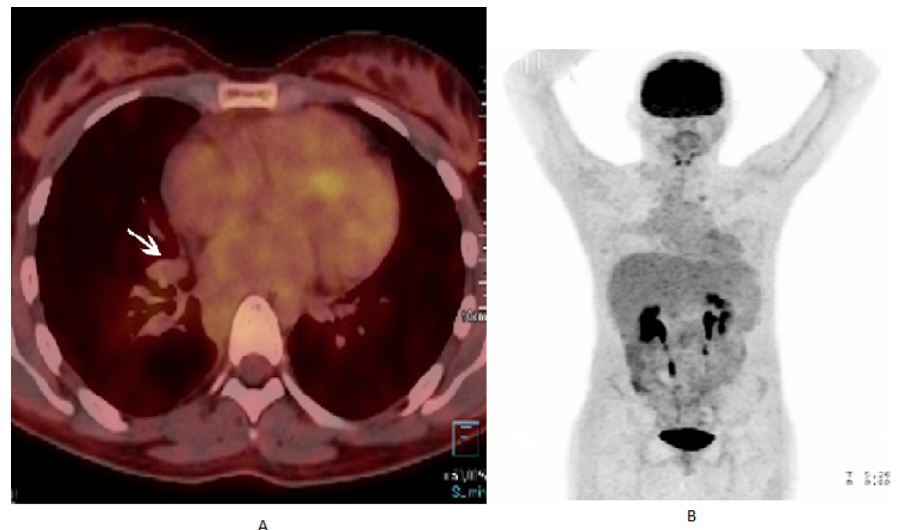

Figure 3. (A) FDG-PET/CT image showing right peri-hilar residual opacity on axial postfusion without anomalous $18 \mathrm{~F}$-fluoro-2-deoxyglucose uptake. Whole body reconstruction (B) also did not reveal other areas of anomalous uptake

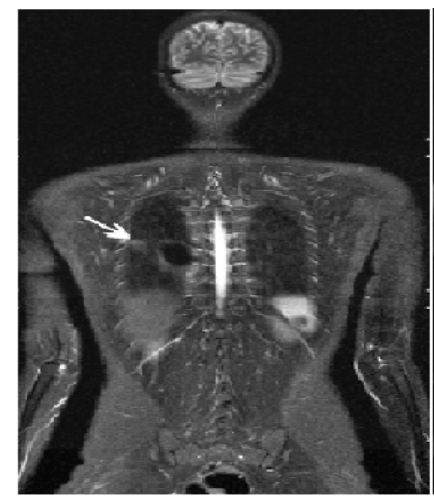

A

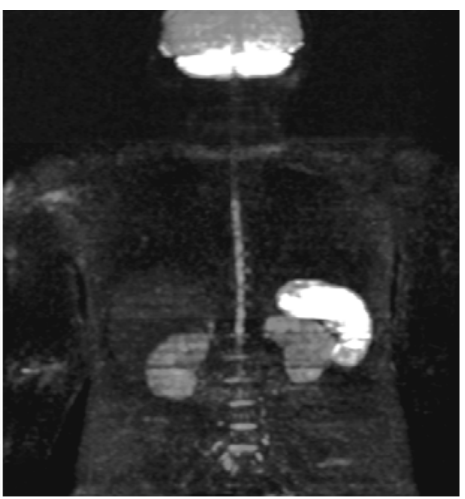

B
Figure 4. (A) Image of WB-MRI/DWI showing residual hyperintense STIR signal in the middle third of the right lung. The MIP DWI reconstruction of the whole body (B) does not show hypersignal, being compatible with residual mass without disease, in agreement with previous FDG-PET/CT

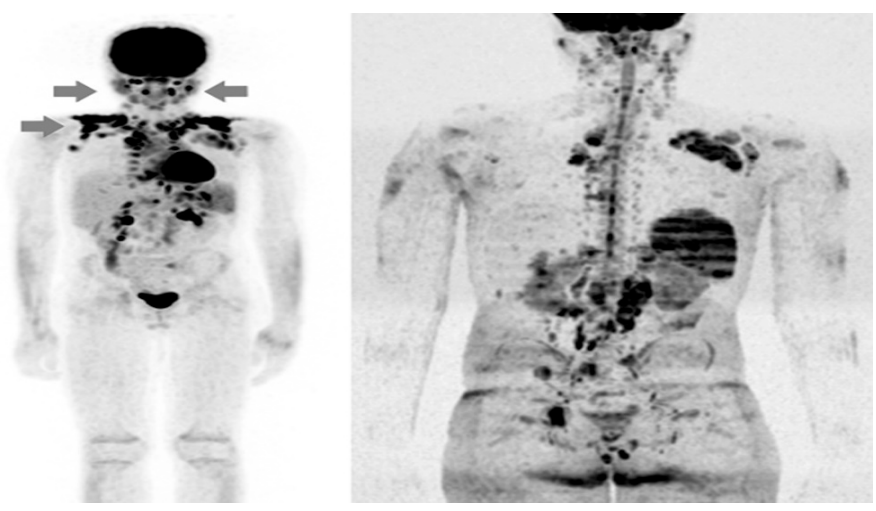

Figure 5. Image from FDG-PET/CT (left) and WB-MRI/DWI (right). Arrows indicating single patient with discrepant sites, present on the first study and not in the WB-MRI/DWI

(above 0.7 ) and a calculated agreement of 95.22\%, Kappa index of 0.8004 .

In post-treatment follow up, both methods performed similar on its findings, with maintenance of a residual CT mass without pathological uptake on FDG-PET/CT (Figures 3A and 3B) or a hyper signal STIR signal without abnormal DWIBS on WB-MRI/DWI (Figures $4 \mathrm{~A}$ and 4B).
All follow-up examinations that had an MRI DWI signal loss occurred were also accompanied by an FDG uptake reduction on FDGPET/CT (Figure 5).

A more significant correlation between those methods was restricted by a greater detection of bone marrow infiltration on WBMRI/DWI during staging examinations, also described in the literature [4,15-19] (Figure 5).

Studies conducted with marrow biopsy as a gold standard for evaluation of bone marrow infiltration showed that WBMRI-DWI did not underestimate any patient, with a higher sensitivity than the conventional methods [16]. That has been assigned for the 27 sites of lesions that were found in the WB-MRI/DWI and not in the FDG-PET/ CT, and clinically followed, with consistent clinical evolution.

It is important to notice that WB-MRI/DWI is an ionizing radiation free study without any related late toxicity, which is known to be as high as younger is the patient exposed [6,7]. Thus, the evaluation of this population by WB-MRI/DWI reduces the risk of second neoplasia from its stochastic effect, attributed to FDG-PET/CT. This risk is remarkable when we treat pathology with high cure rate and survival indexes that can also be exposed to radiotherapy.

In all cases where it was possible to obtain the comparative analysis of the methods in the same patient staging and follow-up, patients $10,14,15$ and 20, there was no follow-up disagreement between the methods in the follow-up examinations.

The ability to detect early response to the treatment without exposure to ionizing radiation is one of the WB-MRI/DWI main advantages, in addition to the possibility to perform multiple scans during patients' treatment, with an early response assessment, without radiations exposure worries, as well as, being more availability on imaging centers $[4,15,19]$.

The latest World Health Organization on European Union members available data from 2014 indicate an availability of 0.61 FDG-PET/CT equipment per 100,000 inhabitants and 1.41 MRI equipment for this same population [34,35]. Newer equipment such as FDG-PET/MRI, which can perform WB-MRI/DWI with the PET component, are even less worldwide available.

The available number of patients retrospectively studied, and consensual exam reading may limit those findings. More expressive data may be obtained in multicenter, prospective, and inter-observer analysis.

\section{Conclusion}

From our data obtained, we concluded that WB-MRI/DWI is a safe and valuable method, with very strong Pearson correlation, and almost perfect Kappa agreement with the FDG-PET/CT gold standard, that can be used as an alternative in the evaluation of children, adolescents and young adults with Hodgkin's lymphoma.

\section{References}

1. Dracham CB, Shankar A, Madan R (2018) Radiation induced secondary malignancies a review article. Radiat Oncol J 36: 85-94. [Crossref]

2. Cheson BD, Fisher RI, Barrington SF, Cavalli F, Schwartz LH, et al. (2014) Recommendations for initial evaluation, staging, and response assessment of Hodgkin and non-Hodgkin lymphoma: the Lugano classification. J Clin Oncol 32: 3059-3068. [Crossref] 
3. Mettler FA Jr, Bhargavan M, Faulkner K, Gilley DB, Gray JE, et al. (2009) Radiologic and nuclear medicine studies in the United States and worldwide: frequency, radiation dose, and comparison with other radiation sources--1950-2007. Radiology 253: 520531. [Crossref]

4. Regacini R, Puchnick A, Shigueoka DC, Iared W, Lederman HM, et al. (2015) Wholebody diffusion-weighted magnetic resonance imaging versus FDG-PET/CT for initial lymphoma staging: systematic review on diagnostic test accuracy studies. Sao Paulo Med J 133: 141-150. [Crossref]

5. Schauer DA, Linton OW (2009) National Council on Radiation Protection and Measurements report substantial medical exposure increase. Radiology 253: 293-296. [Crossref]

6. Brenner DJ, Hall EJ (2007) Computed tomography - an increasing source of radiation exposure. N Engl J Med 357: 2277-2284. [Crossref]

7. Goodman TR, Mustafa A, Rowe E (2019) Pediatric CT radiation exposure: where we were, and where we are now. Pediatr Radiol 49: 469-478. [Crossref]

8. IARC Working Group on the Evaluation of Carcinogenic Risks to Humans (2008) IARC monographs on the evaluation of carcinogenic risks to humans. IARC Monogr Eval Carcinog Risks Hum 97: 3-471.

9. http://www.fda.gov/Radiation-EmittingProducts/RadiationSafety/ RadiationDoseReduction/ucm199994.htm

10. http://www.jointcommission.org/sea_issue_47/

11. Huang B, Law MW, Khong PL (2009) Whole-body PET / CT scanning: estimation of radiation dose and cancer risk. Radiology 251: 166-174. [Crossref]

12. Kwon HW, Kim JP, Lee HJ, Paeng JC, Lee JS, et al. (2016) Radiation Dose from Whole-Body F-18 Fluorodeoxyglucose Positron Emission Tomography/Computed Tomography: Nationwide Survey in Korea. J Korean Med Sci 31: S69-S74. [Crossref]

13. Stejskal EO (1965) Use of spin echoes in a pulsed magnetic-field gradient to study anisotropic, restricted diffusion and flow. J Chem Physics 43: 3597-3603.

14. Attariwala R, Picker W (2013) Whole body MRI: improved lesion detection and characterization with diffusion weighted techniques. J Magn Reson Imaging 38: 253268. [Crossref]

15. Baranska D, Matera K, Podgorski M, Gorska-Chrzastek M, Krajewska K, et al. (2018) Feasibility of diffusion-weighted imaging with DWIBS in staging Hodgkin lymphoma in pediatric patients: comparison with PET/CT. MAGMA 32: 381-3390. [Crossref]

16. Regacini R, Puchnick A, Luisi FAV, Lederman HM (2018) Can diffusion-weighted whole-body MRI replace contrast-enhanced CT for initial staging of Hodgkin lymphoma in children and adolescents?. Pediatr Radiol 48: 638-647. [Crossref]

17. Walker RE, Eustace SJ (2001) Whole-body magnetic resonance imaging: techniques, clinical indications, and future applications. Semin Musculoskelet Radiol 5: 5-20. [Crossref]

18. Gu J, Chan T, Zhang J, Leung AY, Kwong PL, et al. (2011) Whole-body diffusionweighted imaging: the added value to whole-body MRI at initial diagnosis of lymphoma. AJR Am J Roentgenol 197: W384-391. [Crossref]

19. Abdulqadhr G, Molin D, Aström G, Suurkula M, Johansson L, et al. (2011) Wholebody diffusion weighted imaging compared to FDG-PET/CT in staging of lymphoma patients. Acta Radiol 52: 173-180. [Crossref]
20. Kwee TC, Van Ufford HM, Beek FJ, Takahara T, Uiterwaal CS, et al. (2009) Wholebody MRI, including diffusion-weighted imaging, for the initial staging of malignant lymphoma: comparison to computed tomography. Invest Radiol 44: 683-690. [Crossref]

21. Fischer MA, Nanz D, Hany T, Reiner CS, Stolzmann P, et al. (2011) Diagnostic accuracy of whole-body MRI/DWI image fusion for detection of malignant tumors: a comparison with PET/CT. Eur Radiol 21: 246-255. [Crossref]

22. Xu GZ, Li CY, Zhao L, He ZY (2013) Comparison of FDG whole-body PET/CT and gadolinium-enhanced whole-body MRI for distant malignancies in patients with malignant tumors: a meta-analysis. Ann Oncol 24: 96-101. [Crossref]

23. Balbo-Mussetto A, Cirillo S, Bruna R, Gueli A, Saviolo C, et al. (2016) Whole-body MRI with diffusion-weighted imaging: a valuable alternative to contrast-enhanced CT for initial staging of aggressive lymphoma. Clin Radiol 71: 271-279. [Crossref]

24. Kostakoglu L, Evens AM (2014) FDG-PET imaging for Hodgkin lymphoma: curren use and future applications. Clin Adv Hematol Oncol 12: 20-35. [Crossref]

25. Latifoltojar A, Punwani S, Lopes A, Humphries PD, Klusmann M, et al. (2018) 18F-FDG-PET-CT. Full-body MRI for staging and interim response monitoring in pediatric and adolescent Hodgkin's lymphoma: a comparison with multi-modality reference standard including 18F-FDG-PET-CT. Eur Radiol 29: 202-212. [Crossref]

26. Lister TA, Crowther D, Sutcliffe SB, Glatstein E, Canellos GP, et al. (1989) Report of a committee convened to discuss the evaluation and staging of patients with Hodgkin's disease: Cotswolds meeting. J Clin Oncol 7: 1630-1636. [Crossref]

27. Barrington SF, Mikhaeel NG, Kostakoglu L, Meignan M, Hutchings M, et al. (2014) Role of imaging in the staging and response assessment of lymphoma: consensus of the International Conference on Malignant Lymphomas Imaging Working Group. J Clin Oncol 32: 3048-3058. [Crossref]

28. Linet MS, Slovis TL, Miller DL, Kleinerman R, Lee C, et al. (2012) Cancer risks associated with external radiation from diagnostic imaging procedures. $C A$ Cancer J Clin 62: 75-100. [Crossref]

29. Leung DG, Carrino JA, Wagner KR, Jacobs MA (2015) Whole-body magnetic resonance imaging evaluation of facioscapulohumeral muscular dystrophy. Muscle Nerve 52: 512-520. [Crossref]

30. Ahlawat S, Fayad LM, Khan MS, Bredella MA, Harris GJ, et al. (2016) Current wholebody MRI applications in the neurofibromatoses: NF1, NF2, and schwannomatosis. Neurology 87: S31-39. [Crossref]

31. Anupindi SA, Bedoya MA, Lindell RB, Rambhatla SJ, Zelley K, et al. (2015) Diagnostic Performance of Whole-Body MRI as a Tool for Cancer Screening in Children With Genetic Cancer-Predisposing Conditions. AJR Am J Roentgenol 205 400-408. [Crossref]

32. Xu GZ, Li CY, Zhao L, He ZY (2013) Comparison of FDG whole-body PET/CT and gadolinium-enhanced whole-body MRI for distant malignancies in patients with malignant tumors: a meta-analysis. Ann Oncol 24: 96-101. [Crossref]

33. Morone M, Bali MA, Tunariu N, Messiou C, Blackledge M, et al. (2017) WholeBody MRI: Current Applications in Oncology. AJR Am J Roentgenol 209: W336-349. [Crossref]

34. https://gateway.euro.who.int/en/indicators/hlthres_95-magnetic-resonance-imagingunits-per-100-000

35. https://gateway.euro.who.int/en/indicators/hlthres 181-positron-emissiontomography-scanners-per-100-000/

Copyright: (C2019 Schiavon JL. This is an open-access article distributed under the terms of the Creative Commons Attribution License, which permits unrestricted use, distribution, and reproduction in any medium, provided the original author and source are credited. 